\title{
Molecular Imaging of Self-Assembled Rosette Nanotubes by Scanning Tunneling Microscopy
}

\author{
Jae-Young Cho ${ }^{1}$, Liang Shuai ${ }^{2}$, Mounir El-Bakkari ${ }^{2}$, and Hicham Fenniri ${ }^{3}$ \\ 1. National Institute for Nanotechnology, National Research Council (NINT-NRC), 11421 Saskatchewan \\ Drive, Edmonton, Alberta T6G 2M9, Canada \\ 2. Department of Chemistry, University of Alberta, Edmonton, Alberta, CANADA \\ 3. 313 Snell Engineering Center, 360 Huntington Avenue, Northeastern University, Boston, MA 02115 , \\ USA
}

The self-assembled rosette nanotubes (RNT) are organic nanomaterials generated from the selfassembly of a bicyclic guanine-cytosine (GC motif) base. One of the advantages of these nanomaterials is the ability to tailor their function and properties by synthetically modifying the GC motif. Therefore, understanding the detailed supramolecular structure and arrangement of the RNTs with different functional groups is important. The physical characterization of RNTs using microscopy can be challenging because of their nanosclae dimensions ( $c$ a. $3-8 \mathrm{~nm}$ in diameter) and their sensitivity to physical and mechanical damage by standard microscopy techniques. Consequently, proper sample preparation and optimized imaging conditions are critical for successful characterization using microscopy techniques.

\begin{abstract}
Although clear images and measurements of the physical diameters of the RNTs can be obtained using transmission electron microscopy and atomic force microscopy [1-2], imaging the molecular structure of these architectures in greater detail has not been feasible using these characterization tools. The only direct characterization method that can provide details of the RNTs' supramolecular organization is scanning tunneling microscope (STM). In this study, we used ambient STM system since the changes or damages of sample can be minimized compared to ultrahigh vacuum (UHV) STM systems.
\end{abstract}

For the successful STM investigation, a well-defined sharp Pt-Ir tip, evenly dispersed RNTs on the conductive surface of highly ordered pyrolytic graphite (HOPG) and optimized operating conditions of the STM need to be carefully considered. At first, a series of STM tips were fabricated mechanically or/and electrochemically using Pt-Ir wire which is not easily oxidized in air. Then, two different RNTs were deposited using a spin coater on HOPG surface (Figures 1 and 2). Prior to taking an STM image, the sample was first scanned by AFM to ensure the RNTs are evenly dispersed on the HOPG surface. The STM images shown in Figures 1 and 2 were acquired using ambient STM system, a MultiMode Nanoscope IV equipped with an A scanner. The sample surface $(500 \times 500 \mathrm{~nm})$ was scanned using a constant current mode with a low scan rate $(1-2 \mathrm{~Hz})$.

The STM images of the RNTs in Figure 1 clearly show their helical surface structure with rosettes rotating every $20^{\circ}$ along the axis of the tube. Since the calculated inter-rosette distance of this tube is $0.43 \mathrm{~nm}$, every 3 stacks of rosettes show periodic bright spots in the STM image. Interestingly, compared to most of the STM investigations described in the literature, which are done in a UHV STM system with constant height mode [3], the detailed helical molecular structure of the RNTs and 
measurements between the rosette stacks were obtained using a constant current mode (current set point $500 \mathrm{pA}$, bias $1 \mathrm{~V})$ with slow scan rate $(1.97 \mathrm{~Hz})$ in an ambient STM system.

The accurate diameter of the RNT (Figure 2) can be measured by STM along with the details of the supramolecular architecture. Especially, the arrangement of functional groups when two RNTs are bundle together can be observed, as shown in Figure 2c.

In conclusion, this study shows the molecular structures of two different RNTs deposited on HOPG. The STM imaging was carried out with constant current mode under ambient conditions.

\section{References:}

[1] RS Johnson, T Yamazaki, A Kovalenko, and H Fenniri, J. Am. Chem. Soc., 129 (2007), p. 5735.

[2] H Fenniri, B-L Deng, and AE Ribbe, J. Am. Chem. Soc. 124 (2002), p. 11064.

[3] ME. Canas-Ventura, W Xiao, D Wasserfallen, K Mullen, H Brune, JV. Barth, and R Fasel, Angew. Chem. Int. Ed., 46 (2007), p. 1814.

[4] We thank NRC's National Institute for Nanotechnology, University of Alberta, Canada's Natural Science and Engineering Research Council, and Northeastern University for their support.
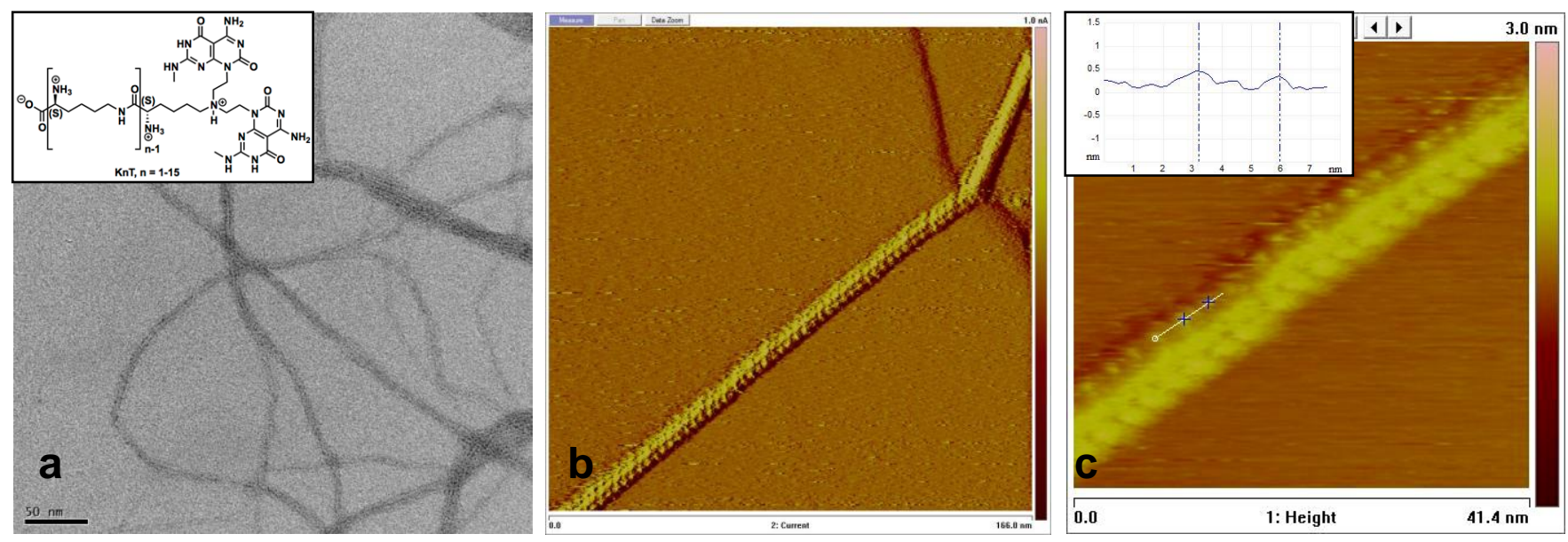

Figure 1. Images of RNTs: a) TEM, b) current mode STM image, c) height mode STM image with height profile.
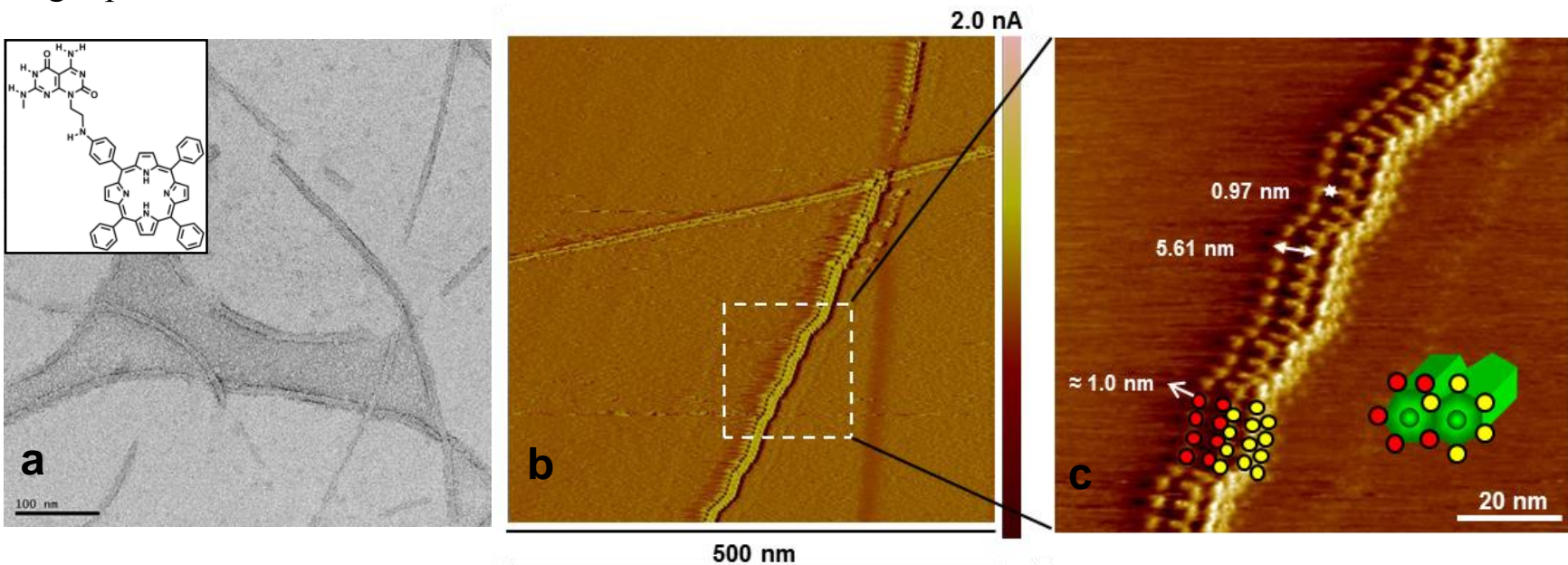

Figure 2. Images of RNTs: a) TEM, b) current mode STM image, c) height mode STM image with schematic diagram. 